\title{
Alta prevalencia en el consumo de tabaco y desigualdades en población Lenca, Intibucá, Honduras, 2015.
}

\author{
High prevalence of tobacco consumption and inequalities in Lenca population, Intibucá, Honduras, 2015.
}

\author{
Nancy Ninoska Arias, ${ }^{1}$ Carlos Mendoza, ${ }^{2}$ Héctor Escalante, ${ }^{3}$ José Tomas Bustillo, ${ }^{4}$ Ana Lucía Mayen. ${ }^{5}$ \\ 1Doctora en Ciencias Químicas y Farmacia, Magister en Salud Pública, Centro Universitario Regional del Centro (CURC-UNAH), Comayagua, Honduras. \\ ${ }^{2} \mathrm{PhD}$ en Bioquímica y Nutrición Humana, Instituto de Nutrición de Centro América y Panamá (INCAP), Ciudad Guatemala, Guatemala. \\ ${ }^{3}$ Doctor en Medicina y Cirugía, Magister en Epidemiologia, PhD en Ciencias del Desarrollo Humano, UNAH, Tegucigalpa, Honduras. \\ ${ }^{4}$ Doctor en Medicina y Cirugía, Master en Razonamiento y Práctica Clínica, Centro de Rehabilitación del Paciente Adicto, Olancho, Honduras. \\ 5Licenciada en Nutrición, PhD en Salud Pública, Organización Mundial de la Salud, Lyon, Francia.
}

RESUMEN. Antecedentes: Aproximadamente un sexto de la población mundial tiene el hábito de fumar para una prevalencia de $24 \%$. La OMS considera que, de no cambiar las tendencias durante el siglo XXI, el tabaco será responsable de la muerte prematura de mil millones de personas. Objetivo: Determinar la prevalencia del consumo de tabaco y la medición de desigualdades socioeconómicas en la población Lenca, Municipio de Intibucá, Intibucá, Honduras, 2015. Métodos: Estudio descriptivo transversal con análisis de asociación. Se aplicó formulario estructurado para recolectar datos generales, sociodemográficos y tabaquismo, previo consentimiento informado. Dependencia al tabaco fue evaluada con test Faguerström. Se prepararon bases de datos en Epi-info-7 y SPSS-18. Se calcularon razones de disparidad (OR). Resultados: De un total de 602 entrevistados, se identificaron 167 fumadores para una prevalencia de consumo de tabaco de $27.7 \%$. El $76.6 \%$ (128) de fumadores tuvo dependencia baja a nicotina y $23.4 \%$ (39) dependencia moderada. La media de ingreso familiar mensual fue USD $\$ 193$ en fumadores y USD $\$ 168$ en no fumadores. El $40.1 \%$ (67) de fumadores gastó mensualmente en tabaco un mínimo de USD $\$ 95$. No se encontró relación entre consumo de tabaco e ingreso familiar mensual (OR=0.87, IC95\% 0.7-1.0) ni con analfabetismo (OR=1.20, IC95\% 0.7-2.0). Discusión: No se observaron diferencias significativas entre consumo de tabaco y las variables socioeconómicas, se encontró una elevada prevalencia de consumo de tabaco en esta población en relación con otros estudios realizados en el país. Los resultados apoyan la necesidad de políticas enfocadas en prevención del tabaco en indígenas.

Palabras clave: Disparidades en el estado de salud, Factores socioeconómicos, Grupos étnicos, Uso de tabaco.

Recibido: 12-03-2019; Aceptado para publicación 30-06-2020 Dirección para correspondencia: Dra. Nancy Ninoska Arias Correo electrónico: ariasninoska23@gmail.com

Declaración de relaciones y actividades financieras y no financieras y conflictos de interés: ninguna.

DOI: https://doi.org/10.5377/rmh.v88i1.11595

\section{INTRODUCCIÓN}

Entre el 2007 y el 2015, la proporción de fumadores de tabaco disminuyó de $23,5 \%$ a $20,7 \% .{ }^{1}$ se espera que esta tendencia continúe y que para el 2025 la prevalencia en la Región baje a $12,9 \%$, aunque las tendencias son alentadoras, es necesario tener presente que todavía uno de cada cinco adultos (15 años o más) consume tabaco en el mundo. ${ }^{1}$ En la Región de las Américas la prevalencia del tabaquismo es $22 \%{ }^{2,3}$ Según OMS gastar más del $10 \%$ del ingreso mensual familiar es una situación que antepone un riesgo de no cumplir las necesidades básicas del grupo familiar. ${ }^{3,4}$

Es imprescindible pensar en las consecuencias del consumo de tabaco y así mismo el gasto en atención médica que esto representa ${ }^{2,5}$ Algunas investigaciones referentes al costo de estas consecuencias, en países como Vietnam, las personas fumadoras gastaron en tabaco 3.6 veces más que en educación y 1.9 veces más que en atención médica en $2003 .{ }^{6}$ En un estudio realizado en Argentina por el Instituto de Efectividad Clínica y Sanitaria (IECS), el costo directo en el sistema de salud atribuible al tabaquismo es USD 33,458,640.699. ${ }^{7}$

Varios estudios han demostrado que la mayor prevalencia del consumo de tabaco se concentra en grupos vulnerables, con bajo nivel socioeconómico o grupos étnicos ${ }^{8,9} \mathrm{La}$ etnia Lenca habita en los departamentos de Intibucá, La Paz, Lempira y el sur de Santa Bárbara; el centro-sur de Francisco Morazán y Valle, colinda con Lencas de El Salvador. En su mayoría son pobres, con poco acceso a los recursos productivos, especialmente a la tierra, su principal fuente de vida y trabajo. Los Lencas de Intibucá tienen como principal actividad productiva y económica la agricultura, alfarería y artesanía. La mujer Lenca es base principal en la economía del pueblo, ya que ella trabaja en la agricultura. ${ }^{10}$ Los Lencas están distribuidos en comunidades constituidas por aldeas, caseríos y pueblos que están localizados en las zonas más altas de Honduras, a más de 1600 metros, zonas que son pocos productivas. ${ }^{11}$ Este estudio se realizó con el objetivo de determinar la prevalencia en el consumo de 
tabaco y la medición de desigualdades socioeconómicas en la población Lenca, Municipio de Intibucá, Honduras, durante el período 2015.

\section{METODOLOGÍA}

Estudio transversal con análisis de asociación. El municipio de Intibucá está conformado por 64 aldeas y 7 caseríos, de los cuales se visitaron a conveniencia 19 aldeas que pertenecen a la etnia Lenca (Figura 1) Los participantes se seleccionaron bajo los siguientes criterios; pertenecientes al grupo étnico Lenca, de cualquier sexo, entre 18 y 65 años de edad y que aceptaron voluntariamente participar en el estudio. No se incluyeron personas que no estaban en el lugar de recolecta de datos durante la entrevista y aquellos que no hablaban español. La muestra se calculó aplicando la formula estadística para población infinita en Excel, se utilizó una proporción esperada del $50 \%$ y un nivel de confianza del $95 \%$ para un tamaño muestral de 600 , el muestreo fue no probabilístico, donde se visitó una de cada dos viviendas de las aldeas seleccionadas, entrevistándose a un miembro de cada casa.

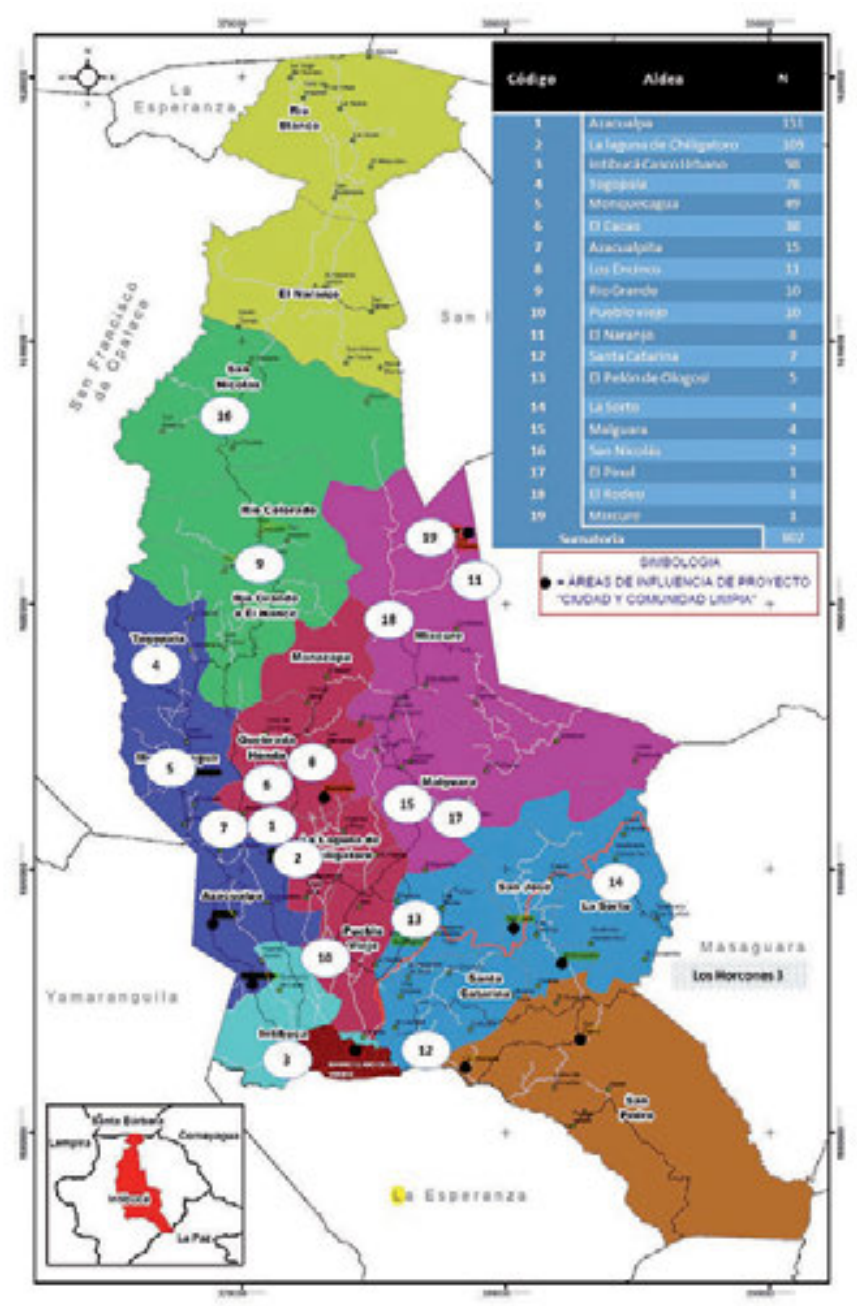

Figura 1. Distribución poblacional por aldeas, consumo de tabaco en la etnia Lenca, Intibucá, Honduras, 2015. Los círculos blancos identifican a las diferentes comunidades de Intibucá, Intibucá, en las que se distribuye la muestra poblacional.
Se utilizó un formulario estructurado para recolectar datos generales, sociodemográficos y tabaquismo. El instrumento fue validado con la participación de 27 residentes no étnicos de Linaca, Tatumbla, Francisco Morazán, aunque esta no es población étnica sirvió para mejorar las preguntas del formulario final. Se obtuvo alfa de Cronbach de 7.08. La dependencia al tabaco fue evaluada con el test Faguerström, que consta de seis preguntas para analizar el nivel de dependencia de nicotina de cigarrillos por medio de una escala: 3 o menos es baja, de 4-6 moderada y de 7 en adelante es alta. ${ }^{12}$ Los formularios fueron aplicados por el investigador principal, el asistente y dos encuestadores a los cuales previamente se les capacitó, usando como técnica la entrevista cara a cara. El trabajo de campo fue supervisado a su vez por el investigador principal y el asistente de investigación.

Se prepararon bases de datos en Epi-info-7 y SPSS-18. Se utilizó razones de disparidad (OR) con su respectivo intervalo de confianza del $95 \%$, las variables utilizadas fueron; consumo de tabaco e ingreso mensual mínimo y máximo de fumadores, consumo de tabaco y sabe leer y escribir para fumadores. En SPSS 18 se construyó un árbol de clasificación para comparar el ingreso familiar mensual de fumadores (este se obtuvo sumando la cantidad que aporta cada miembro y otras fuentes de ingreso de la familia), mismo que se estratifico en ingreso familiar mínimo, medio y máximo de los fumadores con la variable dependiente (consumo de tabaco). Los ingresos son presentados en dólares americanos, a una tasa de cambio para ese momento USD 21.1/ LPS. Para la medición de las desigualdades se empleó la curva de Lorenz, la cual relaciona el índice de Gini, con la renta mensual y la distribución de la población por

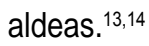

El protocolo fue revisado y aprobado por el Comité de Ética en Investigación Biomédica (CEIB), Facultad de Ciencias Médicas, Universidad Nacional Autónoma de Honduras. A cada participante se le aplicó consentimiento informado, explicándole que su participación era voluntaria, y consistía en contestar una serie de preguntas cuyas respuestas no conllevaban ningún riesgo. La información proporcionada sería utilizada únicamente con fines científicos. Los resultados de este estudio fueron socializados con autoridades municipales del Municipio de Intibucá.

\section{RESULTADOS}

Se entrevistaron 602 personas, $58.1 \%$ (350) del sexo masculino del sexo masculino y $41.9 \%$ (252) del femenino, con edades comprendidas entre 18 y 65 años, procedentes de las aldeas del Municipio de Intibucá que se muestran en la Figura 1. Del total de participantes, se identificaron 167 fumadores, con una prevalencia de consumo de tabaco de $27.7 \%$ (167/602) (IC95\% 24.2-31.3). El 22.9\% de los hombres fumaban o fumaron y $4.8 \%$ de las mujeres para una razón hombre mujer entre los fumadores de 5:1. También se calculó la prevalencia de vida del consumo de tabaco (al menos un familiar que fuma) siendo de 28.7\% (173/602) (IC95\% 25.0-32.4). Las características sociodemográficas de los participantes de acuerdo al consumo de tabaco se presentan en el Cuadro 1. 
Cuadro 1. Características sociodemográficas, consumo de tabaco en la etnia Lenca, Intibucá, Honduras, 2015, n=602.

\begin{tabular}{|c|c|c|c|c|c|c|}
\hline \multirow{2}{*}{ Variable } & \multicolumn{2}{|c|}{ Total } & \multicolumn{2}{|c|}{ No fumador } & \multicolumn{2}{|c|}{ Fumadores } \\
\hline & $\mathrm{N}$ & $(\%)$ & $\mathrm{N}$ & $(\%)$ & $\mathrm{N}$ & $(\%)$ \\
\hline Consumo de Tabaco & 602 & $(100.0)$ & 435 & $(72.3)$ & 167 & $(27.7)$ \\
\hline \multicolumn{7}{|l|}{ Sexo } \\
\hline Mujer & 252 & $(100.0)$ & 223 & $(88.5)$ & 29 & (11.5) \\
\hline Hombre & 350 & $(100.0)$ & 212 & $(60.6)$ & 138 & (39.4) \\
\hline \multicolumn{7}{|l|}{ Grupo etario } \\
\hline $18-33$ & 297 & $(49.3)$ & 216 & $(49.7)$ & 81 & $(48.5)$ \\
\hline $34-49$ & 194 & $(32.2)$ & 144 & $(33.1)$ & 50 & (29.9) \\
\hline $50-65$ & 111 & $(18.4)$ & 75 & $(17.2)$ & 36 & (21.6) \\
\hline \multicolumn{7}{|l|}{ Nivel educativo } \\
\hline Ninguno & 64 & $(10.6)$ & 50 & $(78.1)$ & 14 & $(22.0)$ \\
\hline Primaria & 403 & $(66.9)$ & 289 & $(71.7)$ & 114 & (28.3) \\
\hline Educación Básica & 66 & (11.0) & 55 & $(83.3)$ & 11 & (16.7) \\
\hline Diversificado & 48 & $(8.0)$ & 35 & $(72.9)$ & 13 & $(27.1)$ \\
\hline Técnico & 9 & (1.5) & 7 & $(77.8)$ & 2 & $(22.2)$ \\
\hline Educación Superior & 12 & $(2.0)$ & 9 & $(75.0)$ & 3 & $(25.0)$ \\
\hline \multicolumn{7}{|l|}{ Profesión u oficio } \\
\hline Agricultor & 323 & $(53.6)$ & 201 & $(46.2)$ & 122 & (73.1) \\
\hline Ama de casa & 148 & $(24.6)$ & 132 & $(30.3)$ & 16 & $(9.6)$ \\
\hline Albañil & 13 & $(2.2)$ & 5 & $(1.1)$ & 8 & $(4.8)$ \\
\hline Comerciante Individual & 45 & (7.5) & 38 & $(8.7)$ & 7 & $(4.2)$ \\
\hline Jornalero & 11 & (1.8) & 9 & $(2.1)$ & 2 & $(1.2)$ \\
\hline Maestro (a) & 5 & $(0.8)$ & 3 & $(0.7)$ & 2 & $(1.2)$ \\
\hline Trabajo doméstico & 8 & $(1.3)$ & 8 & $(1.8)$ & 0 & $(0.0)$ \\
\hline Promotor & 1 & $(0.2)$ & 1 & $(0.2)$ & 0 & $(0.0)$ \\
\hline Otro & 48 & (8.0) & 38 & $(8.7)$ & 10 & $(6.0)$ \\
\hline
\end{tabular}

Respecto a la frecuencia de consumo de tabaco, en el momento de la entrevista; el $31.1 \%$ (52) fuma a diario, 23.4\% (39) semanal, $16.2 \%$ (27) mensual y $29.3 \%$ (49) esporádicamente. La mayoría de los entrevistados eran jefes de hogar (53.3\%), conyugue del jefe de hogar (19.8\%), hijos (17.6\%), y la población restante correspondía a padres, nietos, hermanos y otros (nuera, suegro [a], yerno). El gasto mensual de consumo de cigarrillos según nivel de dependencia se muestra en el Cuadro 2. La media de ingreso familiar para fumadores fue de $\$ 193$ mensuales y para los no fumadores fue de $\$ 168$. El $40.1 \%$ (70) de los fumadores realizó un gasto mensual entre \$95-142 en productos de tabaco ( $\geq 49.2 \%$ del ingreso mensual), el $32.3 \%$ (54) tuvo un gasto entre $\$ 0-46$ mensuales ( $\leq 23.8 \%$ del ingreso mensual). El $76.6 \%$ (128) tuvieron dependencia baja y el $23.4 \%$ (39) presento dependencia moderada a la nicotina, no se encontró dependencia alta en este grupo de fumadores. Para este resultado solo se tomó en cuenta los fumadores de cigarrillos, excluyéndose 21 consumidores de tabaco en forma de puros, ya que el test utilizado es específico para cigarrillos.

Se construyó un árbol de clasificación en este caso para medir la estratificación del ingreso con la variable dependiente
Cuadro 2. Gasto mensual de consumo de cigarrillos según nivel de dependencia, consumo de tabaco en la etnia Lenca, Intibucá, Honduras, 2015, n=167.

\begin{tabular}{lccc}
\hline $\begin{array}{l}\text { Gasto mensual en } \\
\text { dólares americanos } \\
\text { en consumo de } \\
\text { tabaco* }\end{array}$ & $\begin{array}{c}\text { Dependencia } \\
\text { Baja } \\
\mathbf{1 - 3} \\
\mathbf{N}\end{array}$ & $\begin{array}{c}\text { Dependencia } \\
\text { Moderada } \\
\mathbf{4}-\mathbf{6}\end{array}$ & $\begin{array}{c}\text { Total } \\
\mathbf{N}\end{array}$ \\
\hline $0-46$ & 26 & 28 & $54(32.3)$ \\
$47-94$ & 25 & 5 & $30(18.0)$ \\
$95-142$ & 67 & 0 & $67(40.1)$ \\
$143-189$ & 4 & 6 & $10(6.0)$ \\
$190-236$ & 6 & 0 & $6(3.6)$ \\
Total & 128 & 39 & $167(100.0)$ \\
\hline${ }^{*}$ El gasto mensual está representado en dólares americanos, tasa de cambio $\$ 1=\mathrm{L} 21.10$
\end{tabular}

(consumo de tabaco), se encontró que el $26.3 \%$ de los fumadores tienen un ingreso menor o igual que $\$ 319.9$, el $37.8 \%$ un ingreso total mayor que $\$ 319.9$. Además, el $16.9 \%$ de los fumadores tiene un ingreso total menor o igual que $\$ 42.8$ (Figura 2). Se 


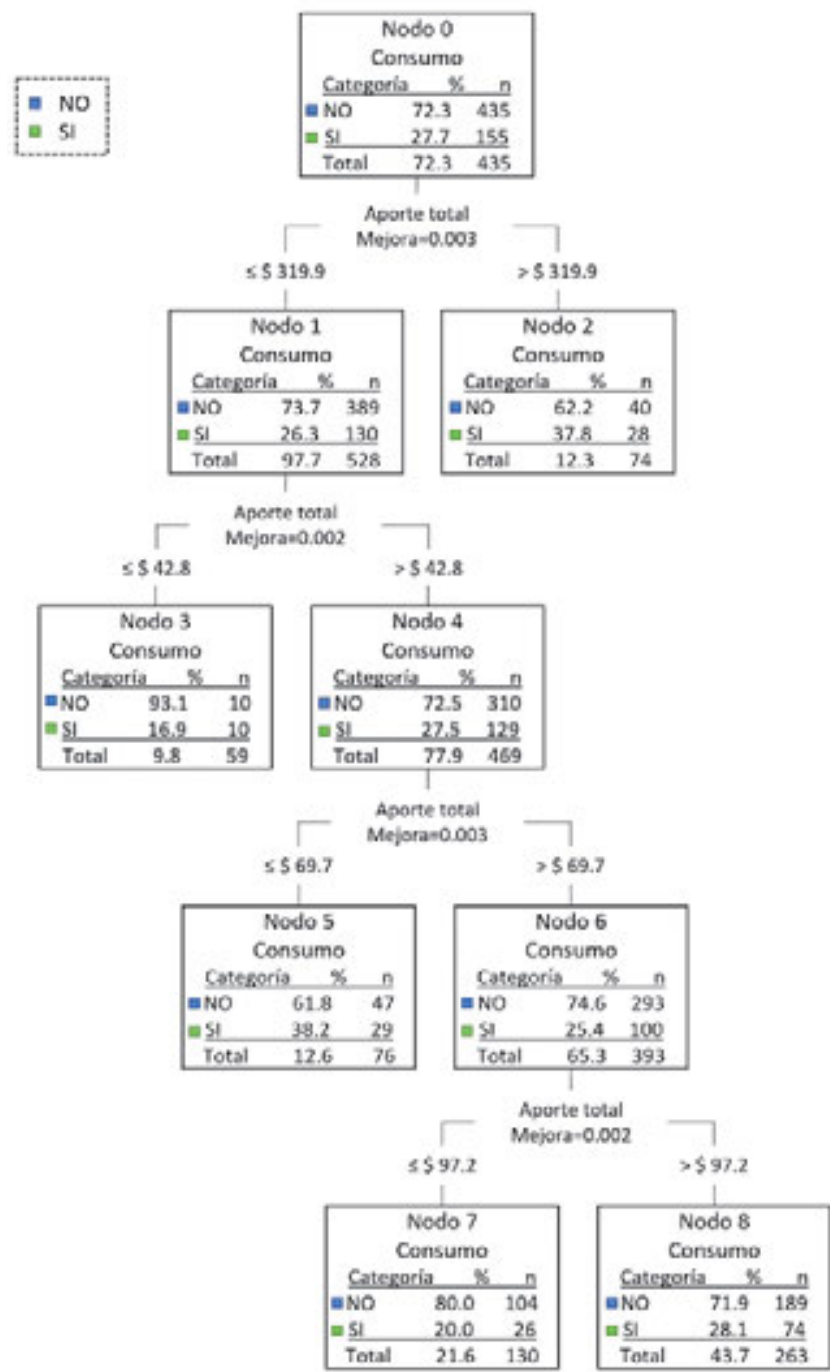

Figura 2. Desigualdad del ingreso entre fumadores y no fumadores, consumo de tabaco en la etnia Lenca, Intibucá, Honduras, 2015. En el árbol de clasificación se agrupa el ingreso económico en nodos, estos representan subgrupos de acuerdo a los márgenes de ingreso específico.

estimó el riesgo del consumo de tabaco en relación al ingreso familiar mensual y este último no se considera factor asociado Odds Ratio=0.87 (IC 95\% 0.7-1.0), así mismo el no saber leer y escribir no es un factor asociado con el consumo tabaco en esta población Odds Ratio=1.20 (IC 95\% 0.7-2.0). Los odss ratio son crudos, no se realizó análisis estratificado o multivariado. Para la desigualdad en la distribución del ingreso total se aplicó la curva de Lorenz donde la población de menor ingreso fue el $15 \%$ obteniendo $10 \%$ del ingreso total, el $60 \%$ representa a la población de ingreso medio el cual obtiene el $50 \%$ del ingreso total y por otra parte la población que tiene mayor ingreso de los encuestados es el $90 \%$ para un ingreso del $80 \%$ del ingreso total (Figura 3).

\section{DISCUSIÓN}

El presente estudio surge a partir de la necesidad de evidencia en el país, relacionado al consumo de tabaco en etnias.

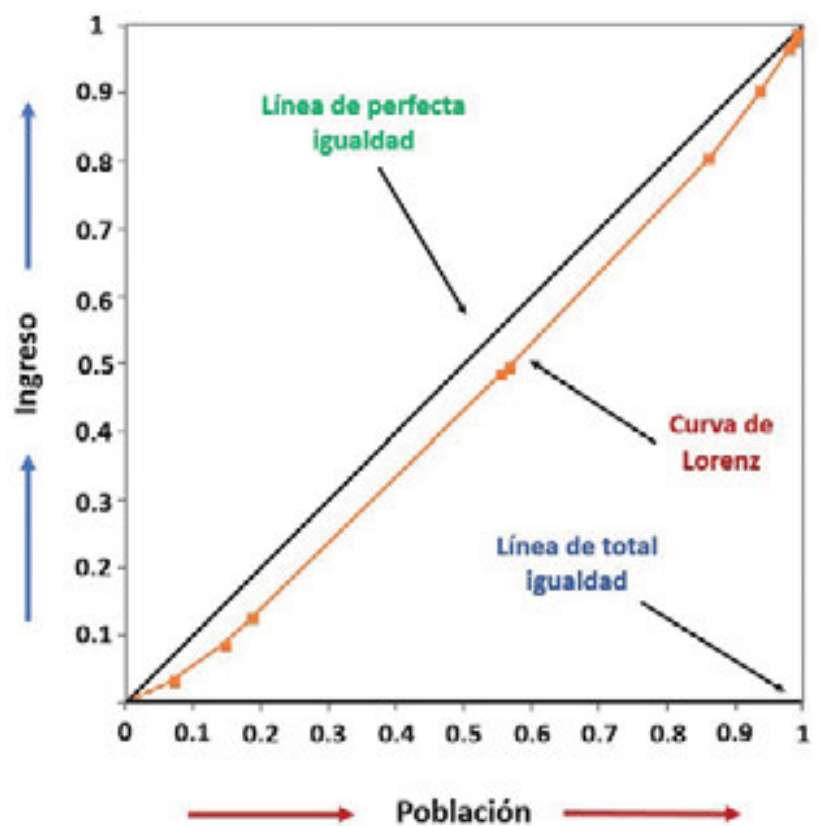

Figura 3. Clasificación socioeconómica de fumadores, consumo de tabaco en la etnia Lenca, Intibucá, Honduras, 2015. La línea naranja muestra la Curva de Lorenz, que representa la distribución del ingreso en la población Lenca, evidenciando la desigualdad del mismo.

A diferencia de otros estudios realizados en Honduras, ${ }^{15}$ no se encontraron diferencias significativas en la asociación entre consumo de tabaco (fumadores y no fumadores) y las variables socioeconómicas, posiblemente debido a un consumo homogéneo en los grupos con distinto nivel socioeconómico. Además, la mayoría de los participantes presentó una dependencia baja a la nicotina.

Se encontró una prevalencia alta de consumo de tabaco en la población estudiada (27.7\%). La cual es 7.3 puntos porcentuales mayor en comparación con la prevalencia nacional que es de $20.4 \%$ y 7.7 puntos porcentuales mayor en comparación con la prevalencia mundial que es de $20 \%$. ${ }^{14,16}$ Por tanto, se puede afirmar que esta población presenta un alto grado de consumo de tabaco en comparación con el resto de la población nacional. Se encontró una gran diferencia de consumo por sexo, en las mujeres representó el $4.8 \%$ de la prevalencia total, pero se observó mayor consumo en mujeres de 50 años en adelante, comparando la prevalencia en mujeres de otros países, un estudio realizado en Karachi, Pakistán, aunque no es una población étnica, el estudio sirve como referencia para comparar el consumo de tabaco en mujeres, la prevalencia de consumo de tabaco fue $18.0 \%$ en mujeres de $15-80$ años de edad (media=37.3 \pm 9.8 ), en relación a estos datos las mujeres Lencas fumaban menos. ${ }^{17}$

En la presente investigación no se encontró asociación entre el consumo de tabaco y el saber leer y escribir, a diferencia de otros estudios sobre desigualdades socioeconómicas, La India reporta que, entre menor nivel educativo e ingreso, existe mayor consumo de tabaco. ${ }^{18}$ También se afirma esta asociación en un estudio realizado en México en población joven, que reporta que la educación $(\mathrm{OR}=2.01 ; 95 \% \mathrm{Cl}$ : 1.03-3.93) está 
altamente asociada al consumo de tabaco ${ }^{19}$ En Nigeria, los estudiantes gastaron alrededor del $40 \%$ de sus ingresos en cigarrillos en 2003, según el informe Atlas del tabaco versión 15.

En diferentes países Latinoamericanos se ha observado la relación entre nivel educativo y consumo de tabaco. En jóvenes de ascendencia mexicana se evaluó el consumo de cigarrillos con el nivel educativo, evidenciándose que los que tenían padres con menor nivel educativo presentaron un mayor riesgo para el consumo de tabaco. ${ }^{19} \mathrm{El}$ consumo de tabaco está asociado con una menor participación presupuestaria asignada a los gastos de salud, educación y vivienda, principalmente para los hogares más pobres. En un estudio realizado en Chile, el no consumir tabaco se relacionó con un mayor gasto en salud: hasta un 32\% para la población total. Asimismo, en el caso de la educación, no consumir tabaco se relaciona estadísticamente con el gasto en educación superior: hasta un 16\% para el total de la población. ${ }^{20}$

Respecto a la dependencia a la nicotina en otros países es elevada en comparación con la población estudiada. En una investigación en México, $82.3 \%$ de fumadores diarios y $98.8 \%$ de ocasionales reportaron dependencia física leve, mientras que $47.9 \%$ y $37.9 \%$, respectivamente, presentaron dependencia psicológica moderada, ${ }^{21}$ en cambio los datos obtenidos en el estudio en la población Lenca, el $76.6 \%$ tuvo dependencia baja y apenas el $23.4 \%$ presento dependencia moderada a la nicotina, ninguno mostro alta dependencia a pesar que se realizó solo en fumadores.

Para la OMS, gastar más del $10 \%$ del ingreso mensual familiar es una situación que antepone un riesgo en no cumplir las necesidades básicas del grupo familiar, ${ }^{3,4}$ en esta población el $40.1 \%$ (70) de los fumadores tiene un gasto mensual entre $\$ 95$ 142 en productos de tabaco ( $\geq 49.2 \%$ del ingreso mensual), el $32.3 \%$ (48) tuvo un gasto entre $\$ 0-46$ mensuales $(\leq 23.8 \%$ del ingreso mensual), con los resultados anteriores, existe el riesgo de que en algunos hogares de esta población no se cumpla con las necesidades básicas familiares.

La principal limitación de este estudio es que, al aplicarse un muestreo no probabilístico a conveniencia, no se logró visitar todas las aldeas, por consiguiente, no se obtuvo una estimación representativa de toda la población, lo cual puede dar lugar a sesgos y con ello, los datos pueden perder validez externa. Sin embargo, es uno de los primeros estudios en evaluar el consu- mo de tabaco en etnias indígenas y su asociación con variables socioeconómicas en el país.

Entre las recomendaciones a nivel local, es que se apliquen medidas para fortalecer la prevención del consumo de tabaco en esta población, a nivel Nacional este estudio se debe considerar para la formulación de políticas públicas para el control del tabaquismo en poblaciones en condiciones de vulnerabilidad y a la vez reforzar la Ley Especial para el Control del Tabaco.

En conclusión, aunque no se encontraron diferencias significativas en la asociación entre consumo de tabaco (fumadores y no fumadores) y las variables socioeconómicas, se obtuvo una elevada prevalencia. Este estudio reveló que esta población Lenca, presenta alto grado de consumo en relación con publicaciones de otros grupos realizados en el país. Los resultados apoyan la necesidad de políticas e intervenciones que se enfoquen en la prevención del tabaco en etnias indígenas.

\section{CONTRIBUCIONES}

NNA, CM, HE, JTB y ALM participaron en la concepción y el diseño del estudio, el análisis e interpretación de los resultados, redacción del artículo y atendieron las recomendaciones editoriales. Todos los autores aprobaron la versión final del artículo. Todos los autores nos hacemos responsables de su contenido.

\section{AGRADECIMIENTOS}

Estamos en deuda con el Centro Internacional de Investigación para el Desarrollo (IDRC), el cual, a través del Instituto de Nutrición para Centroamérica y Panamá, apoyó en cada etapa de este proyecto. A la comunidad de Intibucá y alcalde por permitirnos ingresar a su comunidad para esta investigación, y en especial a cada participante. Agradecemos al equipo de trabajo que contribuyó al éxito de este proyecto: Dra. Ammi Bustillo Ponce, Lic. Mandy Herrera. También estamos agradecidos con la Dra. Francia Ponce y el Dr. Eduardo Fernández por sus aportes brindados. Esperamos que este estudio contribuya a la salud de esta comunidad y de otros. Este estudio fue financiado por el Centro Internacional de Investigación para el Desarrollo (IDRC) de Canadá, a través del Instituto de Nutrición para Centroamérica y Panamá sede Guatemala.

\section{REFERENCIAS}

1. Organización Mundial de la Salud. Informe sobre el control del tabaco en la región de las Américas. Washington DC: OMS; 2018.

2. Fernández Hernández F, Sánchez González E. Coeficiente de inequidad económica del tabaquismo en los servicios de la salud pública. CCM. 2019;23(4):1-10.

3. Organización Mundial de la Salud. MPOWER un plan de medidas para hacer retroceder la epidemia de tabaquismo. Ginebra: OMS; 2008.

4. Organización Mundial de la Salud. Informe OMS sobre la epidemia mundial de tabaquismo, 2009: consecución de ambientes libres de humo de tabaco. Ginebra: OMS; 2009.

5. Organización Panamericana de la Salud. Informe sobre control del tabaco para la Región de las Américas. Washington DC: OPS; 2011.
6. Sriha Belguith A, Bouanene I, Elmhamdi S, Ben Salah A, Harizi C, Soltani $\mathrm{ME}$, et al. Nicotine dependence and carbon monoxide intoxication among adult smokers. Tunis Med. 2015;93(4):231- 6 .

7. Pichon-Riviere A, Augustovski F, Bardach A, Colantonio L. Latinclen Tobacco Research Group. Development and validation of a microsimulation economic model to evaluate the disease burden associated with smoking and the cost-effectiveness of tobacco control interventions in Latin America. Value Health. 2011;14(5 Suppl 1):S51-9.

8. Escalante H. Investigación sobre el alcoholismo y adicciones en la población de los municipios de Intibucá, La Esperanza y Jesús de Otoro, del Departamento de Intibucá, Honduras. Honduras: Comité para la Prevención del Alcoholismo y Drogadicción de Intibucá, Plan Internacional;2011. 
9. González-Bautista E, Zavala-Arciniega L, Rivera-Rivera L, Leyva-López A, Natera-Rey G, Reynales-Shigematsu LM. Factores sociales asociados con el consumo de tabaco y alcohol en adolescentes mexicanos de poblaciones menores a 100000 habitantes. Salud Publica Mex (Cuernavaca). 2019; 61(6):764-74.

10. Von Gleinch U, Gálvez E. Pobreza étnica en Honduras. [Internet]. Washington: BID; 1999 [Consultado abril 2020]. Disponible en: https://publications.iadb.org/es/publicacion/14741/pobreza-etnica-en-honduras

11. Consultores financieros internacionales. Diagnóstico institucional y financiero, municipio de Intibucá [Internet]. Tegucigalpa: BID; 2005 [Consultado abril 2020]. Disponible en: http://www.cofinsa.hn/diag/diaginti.pdf

12. Carrasco Gimenez TJ, Luna Adame M, Vila Castellar J. Validez del Fagerstrom Tolerance Questionnaire como medida de dependencia física de la nicotina: una revisión. Rev Esp Drogodep. 1994;19(1):3-14.

13. Schneider MC, Castillo-Salgado C, Bacallao J, Loyola E, Mujica OJ, Vidaurre M, et al. Métodos de medición de las desigualdades de salud. Rev Panam Salud Pública. 2002;12(6):398-415.

14. Organización Mundial de la Salud. Informe OMS sobre la epidemia mundial de tabaquismo, 2011: advertencia sobre los peligros del tabaco. [Internet]. Ginebra: OMS; 2011 [Consultado abril 2020]. Disponible en: https:/lapps. who.int/iris/bitstream/handle/10665/70681/WHO NMH_TFI_11.3 spa.pdf; isessionid=5196AB9209E7F0CE84978523536A72FF?sequence $=1$

15. Rivera MF. Tabaco y pobreza: un estudio epidemiológico en población urbana. Rev Med Hondur. 2011;79(4):183-6.

16. Eriksen M, Mackay J, Ross H. El Atlas del tabaco. $4^{a}$ ed. Atlanta, GA: Sociedad Americana contra el Cáncer; 2013.

17. Iqbal N, Irfan M, Ashraf N, Awan S, Khan JA. Prevalence of tobacco use among women: a cross sectional survey from a squatter settlement of Karachi, Pakistan. BMC Res Notes. 2015;8: 469.

18. Thakur JS, Prinja S, Bhatnagar N, Rana S, Sinha DN. Socioeconomic inequality in the prevalence of smoking and smokeless tobacco use in India. Asian Pac J Cancer Prev. 2013; 14(11):6965-9

19. Wilkinson AV, Koehly LM, Vandewater EA, Yu RK, Fisher-Hoch SP, Kohl HW, et al. Demographic, psychosocial, and genetic risk associated with smokeless tobacco use among Mexican heritage youth. BMC Med Genet. 2015. 16:43.
20. Paraje G, Araya D. Relationship between smoking and health and education spending in Chile. Tobacco Control. 2018. 27(5):560-567.

21. Paz-Ballesteros WC, Zavala-Arciniega L, Gutiérrez-Torres DS, PoncianoRodríguez G, Reynales-Shigematsu LM. Evaluación de la dependencia física y psicológica al tabaco en fumadores mexicanos adultos, Encodat 2016. Salud Publica Mex. 2019;61(2):136-146

ABSTRACT. Background: Approximately one sixth of the world's population smokes, $24 \%$ prevalence. WHO believes that, by not changing trends, during the 21 st century tobacco will be responsible for the premature death of one billion people. Objective: To determine the prevalence of tobacco consumption and measure socioeconomic inequalities in Lencas, Intibucá, 2015. Methods: Cross-sectional association study. Structured form has been applied to collect general, sociodemographic and smoking data, with prior informed consent. Tobacco dependence was evaluated with Faguerström test. Databases were prepared in Epi-info-7 and SPSS-18. Odds ratios (OR) was calculated. Results: From a total of 602 interviews, 167 smokers were identified with prevalence of $27.7 \%$. The $76.6 \%$ (128) of smokers had low nicotine dependence and $23.4 \%$ (39) remaining moderate. The mean of monthly income was USD $\$ 193$ in smokers and USD $\$ 168$ for not smokers. The $40.1 \%$ (67) of smokers spent at least USD\$95 monthly on tobacco. Relation between tobacco consumption and monthly income were not found $\mathrm{OR}=0.87(95 \% \mathrm{Cl} 0.7-1.0)$ neither with illiteracy $\mathrm{OR}=$ $1.20(95 \% \mathrm{Cl}$ 0.7-2.0). Discussion: No significant differences were observed between tobacco consumption and socioeconomic variables; a high prevalence of consumption was found in this population in relation to other studies carried out in the country. The results support the need for policies focused on tobacco prevention among indigenous people.

Keywords: Ethnic groups, Health status disparities, Socioeconomic factors, Tobacco use. 